\title{
Orbital Stability of Solitary Traveling Waves of Moderate Amplitude
}

\author{
Zhengyong Ouyang \\ Department of Mathematics, Foshan University, Foshan, Guangdong 528000, China \\ Correspondence should be addressed to Zhengyong Ouyang; zyouyang_math@163.com
}

Received 2 December 2014; Accepted 24 January 2015

Academic Editor: Stephen C. Anco

Copyright (C) 2015 Zhengyong Ouyang. This is an open access article distributed under the Creative Commons Attribution License, which permits unrestricted use, distribution, and reproduction in any medium, provided the original work is properly cited.

\begin{abstract}
We consider the orbital stability of solitary traveling wave solutions of an equation describing the free surface waves of moderate amplitude in the shallow water regime. Firstly, we rewrite this equation in Hamiltonian form and construct two invariants of motion. Then using the abstract stability theorem of solitary waves proposed by Grillakis et al. (1987), we prove that the solitary traveling waves of the equation under consideration are orbital stable.
\end{abstract}

\section{Introduction}

In this paper, we consider an equation for surface waves of moderate amplitude in the shallow water regime as follows:

$$
\begin{aligned}
u_{t} & +u_{x}+\frac{3 \alpha}{2} u u_{x}-\frac{3 \alpha^{2}}{8} u^{2} u_{x}+\frac{3 \alpha^{3}}{16} u^{3} u_{x} \\
& +\frac{\beta^{2}}{12}\left(u_{x x x}-u_{x x t}\right)+\frac{7 \alpha \beta^{2}}{24}\left(u u_{x x x}+2 u_{x} u_{x x}\right),
\end{aligned}
$$

where the parameters $\alpha$ and $\beta$ denote amplitude and shallowness. The nonlinear evolution equation (1) arises as an approximation of the Euler equations $[1,2]$. Based on an equation first derived by Johnson [3], on the one hand, one can derive a Camassa-Holm equation at a certain depth below the fluid surface for small amplitude waves [4], on the other hand, for the free surface, a corresponding equation (1) can be derived for waves of moderate amplitude in the shallow water regime. The Camassa-Holm $(\mathrm{CH})$ equation is completely integrable and bi-Hamiltonian, which possesses soliton, peakon, and compacton solutions [5-12], especially breaking waves; that is, the solution remains bounded but its slope becomes unbounded [13]. Besides, the solutions of $\mathrm{CH}$ equation also include global weak solutions [11, 14, 15]. The stability problems of the solutions for the $\mathrm{CH}$ equation and its generalized forms were investigated [16-21], orbital stability of smooth solitary waves, peaked solitary waves and multisolitons were proved. As described the above, many results for waves of small amplitude have been obtained via the $\mathrm{CH}$ equation and its generalized forms. However, it is interesting and necessary to study waves of moderate amplitude. Therefore, the moderate amplitude wave equation (1) was investigated and some results were obtained. For instance, the problems of local well-posedness were considered and the existence and uniqueness of solutions were proved [2]. Only in the special case of parameters $\alpha=4$ and $\beta=\sqrt{12}$, the orbital stability of solitary traveling waves was proved by a method proposed by Grillakis et al. [22, 23]. Nevertheless, the stability problems of solutions for (1) are not solved yet when parameters $\alpha$ and $\beta$ take any values.

In this letter, by a solitary wave we mean a localized traveling wave. The notion of stability is orbital stability, which is the appropriate notion for model equations whose solitary waves are such that the height is proportional to the speed. Indeed, in this case the only type of stability is that of the shape of the wave, a fact that is captured by the notion of orbital stability, as pioneered by considerations made in celestial mechanics. Set a solitary wave $u(x, t)=\varphi(\xi), \xi=$ $x-c t$, where $c>0$ is wave speed. Substituting $u(x, t)=\varphi(\xi)$ into (1), we have

$$
\begin{aligned}
& (1-c) \varphi^{\prime}+\frac{3 \alpha}{2} \varphi \varphi^{\prime}-\frac{3 \alpha^{2}}{8} \varphi^{2} \varphi^{\prime}+\frac{3 \alpha^{3}}{16} \varphi^{3} \varphi^{\prime} \\
& +\frac{\beta^{2}}{12}\left(\varphi^{\prime \prime \prime}+c \varphi^{\prime \prime \prime}\right)+\frac{7 \alpha \beta^{2}}{24}\left(\varphi \varphi^{\prime \prime \prime}+2 \varphi^{\prime} \varphi^{\prime \prime}\right)=0 .
\end{aligned}
$$




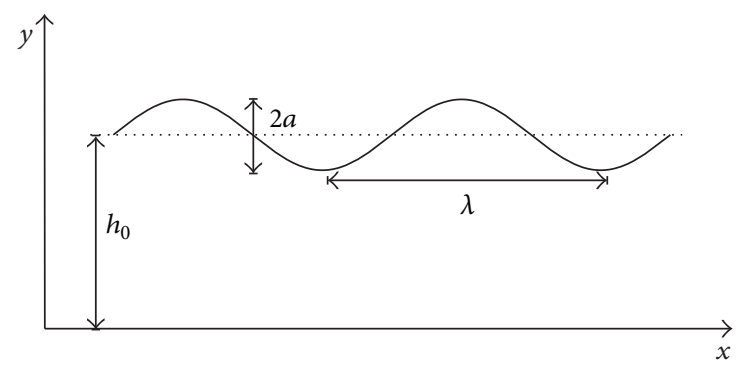

FIGURE 1: Surface traveling wave.

By integration with respect to $\xi$, we obtain from (2) that

$$
\begin{aligned}
c\left(\varphi-\frac{\beta^{2}}{12} \varphi^{\prime \prime}\right)-(\varphi & +\frac{3 \alpha}{4} \varphi^{2}-\frac{\alpha^{2}}{8} \varphi^{3}+\frac{3 \alpha^{3}}{64} \varphi^{4}+\frac{\beta^{2}}{12} \varphi^{\prime \prime} \\
+ & \left.\frac{7 \alpha \beta^{2}}{24}\left(\varphi \varphi^{\prime \prime}+\frac{1}{2}\left(\varphi^{\prime}\right)^{2}\right)\right)=C,
\end{aligned}
$$

where $C$ is an integration constant. Due to the solutions considered which satisfy the property that they are localized and that $\varphi$ and its derivatives decay at infinity, in (3) the constant of integration $C=0$.

\section{Preliminaries}

In (1), the so called amplitude and shallowness parameters $\alpha$ and $\beta$ relate the average length $\lambda$, amplitude $a$, and water depth $h_{0}$ satisfying $\alpha=a / h_{0}>0, \beta=h_{0} / \lambda>0$ (see Figure 1 ).

Let us now give the appropriate notion of solitary waves of (1) and their orbital stability.

The following two quantities $E$ and $F$ are critically important to the proof of the stability:

$$
\begin{gathered}
E(u)=\int_{R}\left(\frac{1}{2} u^{2}+\frac{\beta^{2}}{24} u_{x}^{2}\right) d x \\
F(u)=\int_{R}\left(\frac{1}{2} u^{2}+\frac{\alpha}{4} u^{3}-\frac{\alpha^{2}}{32} u^{4}+\frac{3 \alpha^{3}}{320} u^{5}\right. \\
\left.-\frac{\beta^{2}}{24} u_{x}^{2}-\frac{7 \alpha \beta^{2}}{48} u u_{x}^{2}\right) d x
\end{gathered}
$$

these are constants of the motion; that is, along solutions these expressions are independent of time. The role of these integrals of motion in stability considerations was pioneered in [16].

Definition 1. Let $0 \leqslant T<\infty$. A function $u(x, t) \in C([0, T)$; $\left.H^{2}(R)\right)$ is called a solution to (1) if it satisfies (1) in the distribution sense on $[0, T)$ and the quantities $E(u)$ and $F(u)$ are conserved.

Definition 2. A solitary wave solution $\varphi$ of (1) is called orbital stable if for every $\epsilon>0$ there exists $\delta>0$ such that the following holds: if $u \in\left(C[0, T) ; H^{2}\right)$ is a solution of (1) for some $T \in(0,+\infty)$ with $\left\|u_{0}-\varphi\right\|_{H^{2}}<\delta$, then for every $t \in[0, T)$ we have

$$
\inf _{r \in R}\|u(x, t)-\varphi(\cdot-r)\|_{H^{2}}<\epsilon
$$

Otherwise, the solution is called unstable.

Equation (1) can be rewritten as the following Hamiltonian form:

$$
u_{t}=J F^{\prime}(u)
$$

where $J=-\left(1-(\beta / 12) \partial_{x}^{2}\right)^{-1} \partial_{x}$ is a skew-symmetry operator and $F^{\prime}(u)$ is the Fréchet derivative of $F(u)$.

Using a theorem of Grillakis et al. [22], we deduce the stability problem of solitary waves for (1) from the the convexity of a scalar function, which is relative to the conserved quantities $E(u)$ and $F(u)$. The method used in [22] is described as follows.

Firstly, the following sufficient conditions $(\mathrm{C1})-(\mathrm{C} 3)$ are presented.

(C1) For every $u_{0} \in H^{s}(R), s>3 / 2$, there exists a solution $u$ of $(1)$ in $[0, T)$ such that $u(0)=u_{0}$, where $u \in$ $C\left([0, T) ; H^{s}(R)\right) \cap C^{1}\left([0, T) ; L^{2}(R)\right)$. Furthermore, there exist functionals $E(u)$ and $F(u)$ which are conserved for solutions of (1).

(C2) For every $c \in(1,+\infty)$, there exists a traveling wave solution $\varphi \in H^{2}$ of (1), where $\varphi>0$ and $\varphi_{x} \neq 0$. The mapping $c \mapsto \varphi(x-c t)$ is $C^{1}\left((1,+\infty) ; H^{2}\right)$. Moreover $c E^{\prime}(\varphi)-F^{\prime}(\varphi)=0$, where $E^{\prime}$ and $F^{\prime}$ are the variational derivatives of $E$ and $F$, respectively.

(C3) For every $c \in(1,+\infty)$, the linearized Hamiltonian operator around $\varphi$ defined by

$$
H_{c}: H^{1}(R) \longrightarrow H^{-1}(R), \quad H_{c}=c E^{\prime \prime}(\varphi)-F^{\prime \prime}(\varphi) \text {, }
$$

has exactly one negative simple eigenvalue, its kernel is spanned by $\varphi_{x}$, and the rest of its spectrum is positive and bounded away from zero.

Then under conditions $(\mathrm{C} 1)-(\mathrm{C} 3)$, the relative theorem of stability is as follows.

Theorem 3. If the conditions (C1)-(C3) hold, then solitary waves of (1) are orbital stable if and only if the scalar function

$$
d(c)=c E(\varphi)-F(\varphi)
$$

is convex in a neighborhood of $c$.

\section{Stability of Solitary Waves}

In this section, we show that the conditions (C1)-(C3) of Theorem 3 hold for (1) and then give the result of this paper. 
Lemma 4. The quantities $E(u)$ and $F(u)$ are invariants of motion.

Proof. Multiplying both sides of (1) by $u$ and integrating over the real line, we have

$$
\frac{d}{d t} \int_{R}\left(\frac{1}{2} u^{2}+\frac{\beta^{2}}{24} u_{x}^{2}\right) d x=\frac{d}{d t} E(u(t))=0
$$

proving $E(u)$ is an invariant.

To prove that $F(u)$ is conserved, the Hamiltonian structure of (1) and the skew-symmetry of $J$ is used:

$$
\begin{aligned}
& \frac{d}{d t} F(u(t)) \\
& \quad=\left(F^{\prime}(u), u_{t}\right)=\left(F^{\prime}(u), J F^{\prime}(u)\right)=-\left(J F^{\prime}(u), F^{\prime}(u)\right)
\end{aligned}
$$

that is,

$$
\frac{d}{d t} F(u(t))=0
$$

proving Lemma 4.

For (C1) holds due to Lemma 4 and the existence is proved in [24].

For (C2), by virtue of the existence in [2] and by direct calculation of the variational derivatives of $E$ and $F$, which is

$$
\begin{gathered}
E^{\prime}=u-\frac{\beta^{2}}{12} u_{x x} \\
F^{\prime}=u+\frac{3 \alpha}{4} u^{2}-\frac{\alpha^{2}}{8} u^{3}+\frac{3 \alpha^{3}}{64} u^{4}+\frac{\beta^{2}}{12} u_{x x} \\
+\frac{7 \alpha \beta^{2}}{24}\left(u u_{x x}+\frac{1}{2} u_{x}^{2}\right)
\end{gathered}
$$

(2) can be rewritten as

$$
c E^{\prime}(\varphi)-F^{\prime}(\varphi)=0
$$

proving (C2).

For (C3), by direct calculation, we get the second order variational derivatives of invariants $E(u)$ and $F(u)$ as follows:

$$
\begin{gathered}
E^{\prime \prime}(u)=1-\frac{\beta^{2}}{12} \partial_{x}^{2} \\
F^{\prime \prime}(u)=1+\frac{3 \alpha}{2} u-\frac{3 \alpha^{2}}{8} u^{2}+\frac{3 \alpha^{3}}{16} u^{3}+\frac{\beta^{2}}{12} \partial_{x}^{2} \\
+\frac{7 \alpha \beta^{2}}{24}\left(u_{x x}+u \partial_{x}^{2}+u_{x} \partial_{x}\right) .
\end{gathered}
$$

Substituting $E^{\prime \prime}$ and $F^{\prime \prime}$ into $H_{c}$ we have

$$
\begin{aligned}
H_{c}= & c E^{\prime \prime}(\varphi)-F^{\prime \prime}(\varphi) \\
= & -\left(\frac{\beta^{2}}{12} c+\frac{\beta^{2}}{12}+\frac{7 \alpha \beta^{2}}{24} \varphi\right) \partial_{x}^{2}-\frac{7 \alpha \beta^{2}}{24} \varphi_{x x}-\frac{7 \alpha \beta^{2}}{24} \varphi_{x} \partial_{x} \\
& +c-1-\frac{3 \alpha}{2} \varphi+\frac{3 \alpha^{2}}{8} \varphi^{2}-\frac{3 \alpha^{3}}{16} \varphi^{3} \\
= & -\partial_{x}\left(\left(\frac{\beta^{2}}{12} c+\frac{\beta^{2}}{12}+\frac{7 \alpha \beta^{2}}{24} \varphi\right) \partial_{x}\right)+c-1-\frac{3 \alpha}{2} \varphi \\
& +\frac{3 \alpha^{2}}{8} \varphi^{2}-\frac{3 \alpha^{3}}{16} \varphi^{3}-\frac{7 \alpha \beta^{2}}{24} \varphi_{x x} .
\end{aligned}
$$

In order to prove (C3) holds, we consider the spectral problem of $H_{c}$. We show that, for every $c>1$, the operator $H_{c}$ has exactly one negative simple eigenvalue while the rest of the spectrum is positive and bounded away from zero.

The spectral equation $H_{c} v=\lambda v$ can be transformed to the Sturm-Liouville problem

$$
-\left(p v_{x}\right)_{x}+(q-\lambda) v=0
$$

where $p=\left(\beta^{2} / 12\right) c+\beta^{2} / 12+\left(7 \alpha \beta^{2} / 24\right) \varphi, q=c-1-$ $(3 \alpha / 2) \varphi+\left(3 \alpha^{2} / 8\right) \varphi^{2}-\left(3 \alpha^{3} / 16\right) \varphi^{3}-\left(7 \alpha \beta^{2} / 24\right) \varphi_{x x}$.

The linearized Hamiltonian operator $H_{c}$ is a self-adjoint, second order differential operator. So its eigenvalues $\lambda$ are real and simple, and its essential spectrum is given by $[c-1, \infty)$ by virtue of the fact that $\liminf _{x \rightarrow \infty} q(x)=c-1$. Moreover, it can be directly shown that (2) is equivalent to $H_{c}\left(\varphi_{x}\right)=0$. Due to the fact that the solitary wave solutions of (1) have a unique maximum, $\varphi_{x}$ has only one zero. By Sturm-Liouville Theorem, zero is the second eigenvalue of $H_{c}$, the operator $H_{c}$ has exactly one negative simple eigenvalue, and the rest of the spectrum is positive and bounded away from zero.

Under the conditions $(\mathrm{C} 1)-(\mathrm{C} 3)$, the stability problem of solitary waves for (1) can be transformed to the problem of convex of the scalar function $d(c)$. We have the following theorem.

Theorem 5. For each wave speed $c>1$, the scalar function $d(c)$ is convex in a neighborhood of $c$. Therefore, all solitary wave solutions of (1) are orbital stable.

\section{Proof of Stability}

Equation (3) can be rewritten as the following plane system:

$$
\begin{aligned}
\varphi^{\prime}=y, \\
y^{\prime}=\left(c \varphi-\varphi-\frac{3 \alpha}{4} \varphi^{2}+\frac{\alpha^{2}}{8} \varphi^{3}-\frac{3 \alpha^{3}}{64} \varphi^{4}-\frac{7 \alpha \beta^{2}}{48} y^{2}\right) \\
\cdot\left(c+\frac{\beta^{2}}{12}+\frac{7 \alpha \beta^{2}}{24} \varphi\right)^{-1} .
\end{aligned}
$$


The first integral of the above plane system is

$$
\begin{aligned}
H(\varphi, y)= & c \varphi^{2}-\varphi^{2}-\frac{\alpha}{2} \varphi^{3}+\frac{\alpha^{2}}{16} \varphi^{4}-\frac{3 \alpha^{3}}{160} \varphi^{5} \\
& -\left(c+\frac{\beta^{2}}{12}\right) y^{2}+\frac{7 \alpha \beta^{2}}{24} \varphi y^{2}=h,
\end{aligned}
$$

where $h$ is the integral constant. Since $\varphi \rightarrow 0, \varphi^{\prime}$ (or $\left.y\right) \rightarrow 0$ as $\xi \rightarrow \infty$, so the integral constant $h=0$. From (18), we can get that solitary traveling wave solutions $\varphi$ of (1) satisfy

$$
\varphi^{\prime 2}=y^{2}=\frac{c-1-(\alpha / 2) \varphi+\left(\alpha^{2} / 16\right) \varphi^{2}-\left(3 \alpha^{3} / 160\right) \varphi^{3}}{c+\beta^{2} / 12+\left(7 \alpha \beta^{2} / 24\right) \varphi} \varphi^{2}
$$

Due to the symmetry of solitary wave solutions $\varphi$ with respect to the crest, on $(0,+\infty)$ we have $\varphi^{\prime}<0$ and

$$
\begin{aligned}
\varphi^{\prime} & =-\varphi \sqrt{\frac{c-1-(\alpha / 2) \varphi+\left(\alpha^{2} / 16\right) \varphi^{2}-\left(3 \alpha^{3} / 160\right) \varphi^{3}}{c+\beta^{2} / 12+\left(7 \alpha \beta^{2} / 24\right) \varphi}} \\
& =-\varphi \sqrt{\frac{f_{2}(\varphi, c)}{f_{1}(\varphi, c)}},
\end{aligned}
$$

where $f_{1}(\varphi, c)=c+\beta^{2} / 12+\left(7 \alpha \beta^{2} / 24\right) \varphi$ and $f_{2}(\varphi, c)=c-$ $1-(\alpha / 2) \varphi+\left(\alpha^{2} / 16\right) \varphi^{2}-\left(3 \alpha^{3} / 160\right) \varphi^{3}$.

Lemma 6. Let $f_{2}(\varphi, c)=c-1-(\alpha / 2) \varphi+\left(\alpha^{2} / 16\right) \varphi^{2}-$ $\left(3 \alpha^{3} / 160\right) \varphi^{3}$ and let $M(c)$ denote the maximum of $\varphi$; then $f_{2}(\cdot, c)$ has only one zero point $\varphi=M(c)$ for each wave speed $c$.

Proof. For each given wave speed $c$, differentiating $f_{2}(\varphi, c)$ with respect to $\varphi$ yields

$$
\begin{aligned}
\frac{\partial}{\partial \varphi} f_{2}(\varphi, c) & =-\frac{9 \alpha^{3}}{160} \varphi^{2}+\frac{\alpha^{2}}{8} \varphi-\frac{\alpha}{2} \\
& =\alpha\left(-\frac{9}{160}\left(\alpha \varphi-\frac{10}{9}\right)^{2}-\frac{31}{72}\right)<0 ;
\end{aligned}
$$

thus $f_{2}(\cdot, c)$ is monotonic. Moreover, for any given wave speed $c$, we have $\varphi^{\prime}=0$ when $\varphi$ takes the maximum $M(c)$. It is implied from (19) that

$$
c-1-\frac{\alpha}{2} M(c)+\frac{\alpha^{2}}{16} M(c)^{2}-\frac{3 \alpha^{3}}{160} M(c)^{3}=0
$$

namely, $f_{2}(M(c), c)=0$. Lemma 6 is proved.
By direct calculation, we have

$$
\begin{aligned}
d^{\prime}(c) & =E(\varphi)+c E^{\prime}(\varphi)-F^{\prime}(\varphi)=E(\varphi) \\
& =\int_{R}\left(\frac{1}{2} \varphi^{2}+\frac{\beta^{2}}{24} \varphi_{x}^{2}\right) d x=\int_{0}^{\infty}\left(\varphi^{2}+\frac{\beta^{2}}{12} \varphi_{x}^{2}\right) d x \\
& =\int_{0}^{\infty} \varphi^{2}\left(1+\frac{\beta^{2}}{12} \frac{f_{2}(\varphi, c)}{f_{1}(\varphi, c)}\right) d x \\
& =-\int_{0}^{\infty} \varphi \varphi^{\prime} \sqrt{\frac{f_{1}(\varphi, c)}{f_{2}(\varphi, c)}\left(1+\frac{f_{2}(\varphi, c)}{f_{1}(\varphi, c)}\right) d x} \\
& =-\int_{0}^{\infty} \varphi \varphi^{\prime} \frac{12 f_{1}(\varphi, c)+\beta^{2} f_{2}(\varphi, c)}{12 \sqrt{f_{1}(\varphi, c) f_{2}(\varphi, c)}} d x \\
& =\int_{0}^{M(c)} \eta \frac{12 f_{1}(\eta, c)+\beta^{2} f_{2}(\eta, c)}{12 \sqrt{f_{1}(\eta, c) f_{2}(\eta, c)}} d \eta .
\end{aligned}
$$

In the last equality, we use the transformation $\varphi(x)=\eta$ and employ the fact that $\varphi(x)$ takes a unique maximum $M(c)$, which corresponds to the unique real root of $f_{2}(\varphi, c)$ in Lemma 6. Unfortunately $f_{2}(M(c), c)=0$ precludes a straightforward differentiation of $d^{\prime}(c)$. To prove $d^{\prime \prime}(c)>0$, another way is to show the existence of $d^{\prime \prime}(c)$ and then $d^{\prime}(c)$ is an increasing function.

Lemma 7. For any wave speed $c>1$, the derivative of $d^{\prime}(c)$ exists.

Proof. It was presented in [2] that the function $M(c)$ : $[1, \infty) \rightarrow[0, \infty)$ is bijective and strictly monotonically increasing; $M(1)=0$ and $\lim _{c \rightarrow \infty} M(c)=\infty$. Let $H=M(c)$; by (22) we have

$$
c=M^{-1}(H)=1+\frac{\alpha}{2} H-\frac{\alpha^{2}}{16} H^{2}+\frac{3 \alpha^{3}}{160} H^{3} .
$$

Substituting $H=M(c)$ and $C=M^{-1}(H)$ into (23) yields

$d^{\prime}(c)$

$$
\begin{aligned}
& =\int_{0}^{H} \eta \frac{12 f_{1}\left(\eta, M^{-1}(H)\right)+\beta^{2} f_{2}\left(\eta, M^{-1}(H)\right)}{12 \sqrt{f_{1}\left(\eta, M^{-1}(H)\right) f_{2}\left(\eta, M^{-1}(H)\right)}} d \eta \\
& =\int_{0}^{1} H^{2} \tau \frac{12 f_{1}\left(H \tau, M^{-1}(H)\right)+\beta^{2} f_{2}\left(H \tau, M^{-1}(H)\right)}{12 \sqrt{f_{1}\left(H \tau, M^{-1}(H)\right) f_{2}\left(H \tau, M^{-1}(H)\right)}} d \tau,
\end{aligned}
$$

where we have made a transformation $\eta=H \tau$. The integrand in the above integral is singular only when $\tau=1$ according to the transformation made; thus the integral is well defined on $(0,1)$. 
Let $f(\tau, H)$ denote the integrand in (25). For any interval $\left[H_{1}, H_{2}\right]$ with $H_{1}>0, \int_{0}^{1} f(\tau, H) d \tau$ can be regarded as a parameter integral. It is observed that $f(\cdot, H) \in L^{1}[0,1]$ for all $H \in\left[H_{1}, H_{2}\right]$ and $f(\tau, \cdot) \in C^{1}\left[H_{1}, H_{2}\right]$ for all $\tau \in(0,1)$. By direct calculation, we have

$$
\partial_{H} f(\tau, H)=\frac{H^{2} \tau(1-\tau) N(\tau, H, \alpha, \beta)}{\left(f_{1}\left(\tau H, M^{-1} H\right) f_{2}\left(\tau H, M^{-1} H\right)\right)^{3 / 2}},
$$

where

$$
\begin{aligned}
& f_{1}\left(\tau H, M^{-1} H\right) \\
& \quad=1+\frac{\beta^{2}}{12}+\frac{\alpha}{2} H-\frac{\alpha^{2}}{16} H^{2}+\frac{3 \alpha^{3}}{160} H^{3}+\frac{7 \alpha \beta^{2}}{24} H \tau, \\
& f_{2}\left(\tau H, M^{-1} H\right) \\
& =H(1-\tau)\left(\frac{\alpha}{2}-\frac{\alpha^{2}}{16} H(1+\tau)+\frac{3 \alpha^{3}}{160} H^{2}\left(1+\tau+\tau^{2}\right)\right),
\end{aligned}
$$

and the expression of $N(\tau, H, \alpha, \beta)$ can be shown as follows:

$N(\tau, H, \alpha, \beta)$

$$
\begin{aligned}
&=\frac{1}{256000} \\
& \cdot\left(\alpha \left(110592000+119808000 H \alpha+8294400 H^{2} \alpha^{2}\right.\right. \\
& \quad-3801600 H^{3} \alpha^{3}+4924800 H^{4} \alpha^{4}-979200 H^{5} \alpha^{5} \\
&+ 220320 H^{6} \alpha^{6}-19440 H^{7} \alpha^{7}+1944 H^{8} \alpha^{8} \\
&+18432000 \beta^{2}+16896000 H \alpha \beta^{2}-1305600 H^{2} \alpha^{2} \beta^{2} \\
&+518400 H^{3} \alpha^{3} \beta^{2}+316800 H^{4} \alpha^{4} \beta^{2}-66480 H^{5} \alpha^{5} \beta^{2} \\
&+18360 H^{6} \alpha^{6} \beta^{2}-1620 H^{7} \alpha^{7} \beta^{2}+162 H^{8} \alpha^{8} \beta^{2} \\
&+768000 \beta^{4}+576000 H \alpha \beta^{4}-166400 H^{2} \alpha^{2} \beta^{4} \\
&+69600 H^{3} \alpha^{3} \beta^{4}-7800 H^{4} \alpha^{4} \beta^{4}+1260 H^{5} \alpha^{5} \beta^{4} \\
&-9216000 H \alpha \tau-10137600 H^{2} \alpha^{2} \tau+345600 H^{3} \alpha^{3} \tau \\
&+748800 H^{4} \alpha^{4} \tau-504000 H^{5} \alpha^{5} \tau+142560 H^{6} \alpha^{6} \tau \\
&-19440 H^{7} \alpha^{7} \tau+1944 H^{8} \alpha^{8} \tau+66048000 H \alpha \beta^{2} \tau \\
&+32486400 H^{2} \alpha^{2} \beta^{2} \tau-8121600 H^{3} \alpha^{3} \beta^{2} \tau \\
&+3240000 H^{4} \alpha^{4} \beta^{2} \tau-347280 H^{5} \alpha^{5} \beta^{2} \tau \\
&+ 57240 H^{6} \alpha^{6} \beta^{2} \tau-1620 H^{7} \alpha^{7} \beta^{2} \tau+162 H^{8} \alpha^{8} \beta^{2} \tau \\
&+ 5568000 H \alpha \beta^{4} \tau+1241600 H^{2} \alpha^{2} \beta^{4} \tau
\end{aligned}
$$

$-391200 H^{3} \alpha^{3} \beta^{4} \tau+195200 H^{4} \alpha^{4} \beta^{4} \tau$

$-21840 H^{5} \alpha^{5} \beta^{4} \tau+3780 H^{6} \alpha^{6} \beta^{4} \tau$

$+1382400 H^{2} \alpha^{2} \tau^{2}+2073600 H^{3} \alpha^{3} \tau^{2}$

$+345600 H^{4} \alpha^{4} \tau^{2}-86400 H^{5} \alpha^{5} \tau^{2}$

$+93960 H^{6} \alpha^{6} \tau^{2}-11340 H^{7} \alpha^{7} \tau^{2}$

$+1944 H^{8} \alpha^{8} \tau^{2}-4377600 H^{2} \alpha^{2} \beta^{2} \tau^{2}$

$-1929600 H^{3} \alpha^{3} \beta^{2} \tau^{2}+1221600 H^{4} \alpha^{4} \beta^{2} \tau^{2}$

$-277680 H^{5} \alpha^{5} \beta^{2} \tau^{2}+50490 H^{6} \alpha^{6} \beta^{2} \tau^{2}$

$-675 H^{7} \alpha^{7} \beta^{2} \tau^{2}+162 H^{8} \alpha^{8} \beta^{2} \tau^{2}$

$+10377600 H^{2} \alpha^{2} \beta^{4} \tau^{2}-1087200 H^{3} \alpha^{3} \beta^{4} \tau^{2}$

$+270200 H^{4} \alpha^{4} \beta^{4} \tau^{2}-21840 H^{5} \alpha^{5} \beta^{4} \tau^{2}$

$+3780 H^{6} \alpha^{6} \beta^{4} \tau^{2}+374400 H^{3} \alpha^{3} \beta^{2} \tau^{3}$

$+552000 H^{4} \alpha^{4} \beta^{2} \tau^{3}-66720 H^{5} \alpha^{5} \beta^{2} \tau^{3}$

$+27000 H^{6} \alpha^{6} \beta^{2} \tau^{3}+1620 H^{7} \alpha^{7} \beta^{2} \tau^{3}$

$-162 H^{8} \alpha^{8} \beta^{2} \tau^{3}-640800 H^{3} \alpha^{3} \beta^{4} \tau^{3}$

$+208000 H^{4} \alpha^{4} \beta^{4} \tau^{3}-1260 H^{5} \alpha^{5} \beta^{4} \tau^{3}$

$+3780 H^{6} \alpha^{6} \beta^{4} \tau^{3}+93600 H^{4} \alpha^{4} \beta^{2} \tau^{4}$

$+24480 H^{5} \alpha^{5} \beta^{2} \tau^{4}-10530 H^{6} \alpha^{6} \beta^{2} \tau^{4}$

$+1620 H^{7} \alpha^{7} \beta^{2} \tau^{4}-162 H^{8} \alpha^{8} \beta^{2} \tau^{4}$

$+40000 H^{4} \alpha^{4} \beta^{4} \tau^{4}+21840 H^{5} \alpha^{5} \beta^{4} \tau^{4}$

$-3780 H^{6} \alpha^{6} \beta^{4} \tau^{4}-15120 H^{5} \alpha^{5} \beta^{2} \tau^{5}$

$-6480 H^{6} \alpha^{6} \beta^{2} \tau^{5}+675 H^{7} \alpha^{7} \beta^{2} \tau^{5}-162 H^{8} \alpha^{8} \beta^{2} \tau^{5}$

$+21840 H^{5} \alpha^{5} \beta^{4} \tau^{5}-3780 H^{6} \alpha^{6} \beta^{4} \tau^{5}$

$\left.\left.-3780 H^{6} \alpha^{6} \beta^{4} \tau^{6}\right)\right)$.

Therefore, there exists a positive constant $K$ related to $\alpha$, $\beta$ and $\left[H_{1}, H_{2}\right]$ such that

$$
\left|\partial_{H} f(\tau, H)\right| \leq K(1-\tau)^{-1 / 2} \quad \forall(\tau, H) \in(0,1) \times\left[H_{1}, H_{2}\right] .
$$

Denote $g(\tau)=K(1-\tau)^{-1 / 2}$; then $g(\tau) \in L^{1}(0,1)$. By the theorem on differentiation of parameter integrals, we have

$$
\partial_{H} \int_{0}^{1} f(\tau, H) d \tau=\int_{0}^{1} \partial_{H} f(\tau, H) d \tau
$$


note that $f(\tau, \cdot) \in C^{1}\left[H_{1}, H_{2}\right]$ for all $\tau \in(0,1)$, by virtue of the arbitrary of $H_{1}$ and $H_{2}$, which ensures the existence of derivative of $d^{\prime}(c)$ and

$$
d^{\prime \prime}(c)=\int_{0}^{1} \partial_{H} f(\tau, H) d \tau \cdot M^{\prime}(c)
$$

Lemma 7 is proved.

It is not easy to prove $d^{\prime \prime}(c)>0$ directly, so we turn to show the following Lemma.

Lemma 8. For wave speed $c>1, d^{\prime}(c)$ is an increasing function with respect to $c$.

Proof. The function $M(c):[1, \infty) \rightarrow[0, \infty)$ is bijective and strictly monotonically increasing [2]; therefore, for any wave speeds $c_{2}>c_{1}>1$, the corresponding height of waves $M\left(c_{2}\right)>M\left(c_{1}\right)$, we show that $d^{\prime}\left(c_{2}\right)-d^{\prime}\left(c_{1}\right)>0$.

$$
\begin{aligned}
& d^{\prime}\left(c_{2}\right)-d^{\prime}\left(c_{1}\right) \\
& =\int_{0}^{M\left(c_{2}\right)} \eta \frac{12 f_{1}\left(\eta, c_{2}\right)+\beta^{2} f_{2}\left(\eta, c_{2}\right)}{12 \sqrt{f_{1}\left(\eta, c_{2}\right) f_{2}\left(\eta, c_{2}\right)}} d \eta \\
& -\int_{0}^{M\left(c_{1}\right)} \eta \frac{12 f_{1}\left(\eta, c_{1}\right)+\beta^{2} f_{2}\left(\eta, c_{1}\right)}{12 \sqrt{f_{1}\left(\eta, c_{1}\right) f_{2}\left(\eta, c_{1}\right)}} d \eta \\
& \geq \int_{0}^{M\left(c_{1}\right)} \eta \frac{12 f_{1}\left(\eta, c_{2}\right)+\beta^{2} f_{2}\left(\eta, c_{2}\right)}{12 \sqrt{f_{1}\left(\eta, c_{2}\right) f_{2}\left(\eta, c_{2}\right)}} d \eta \\
& -\int_{0}^{M\left(c_{1}\right)} \eta \frac{12 f_{1}\left(\eta, c_{1}\right)+\beta^{2} f_{2}\left(\eta, c_{1}\right)}{12 \sqrt{f_{1}\left(\eta, c_{1}\right) f_{2}\left(\eta, c_{1}\right)}} d \eta \\
& =\int_{0}^{M\left(c_{1}\right)} \eta\left(\frac{12 f_{1}\left(\eta, c_{2}\right)+\beta^{2} f_{2}\left(\eta, c_{2}\right)}{12 \sqrt{f_{1}\left(\eta, c_{2}\right) f_{2}\left(\eta, c_{2}\right)}}\right. \\
& \left.-\frac{12 f_{1}\left(\eta, c_{1}\right)+\beta^{2} f_{2}\left(\eta, c_{1}\right)}{12 \sqrt{f_{1}\left(\eta, c_{1}\right) f_{2}\left(\eta, c_{1}\right)}}\right) d \eta \\
& \geq \int_{0}^{M\left(c_{1}\right)} \eta\left(\frac{12 f_{1}\left(\eta, c_{2}\right)+\beta^{2} f_{2}\left(\eta, c_{2}\right)}{12 \sqrt{f_{1}\left(\eta, c_{2}\right) f_{2}\left(\eta, c_{2}\right)}}\right. \\
& \left.-\frac{12 f_{1}\left(\eta, c_{1}\right)+\beta^{2} f_{2}\left(\eta, c_{1}\right)}{12 \sqrt{f_{1}\left(\eta, c_{2}\right) f_{2}\left(\eta, c_{2}\right)}}\right) d \eta
\end{aligned}
$$

$$
\begin{aligned}
\int_{0}^{M\left(c_{1}\right)} \eta( & 12\left(f_{1}\left(\eta, c_{2}\right)-f_{1}\left(\eta, c_{1}\right)\right) \\
& \left.+\beta^{2}\left(f_{2}\left(\eta, c_{2}\right)-f_{2}\left(\eta, c_{1}\right)\right)\right) \\
& \left.\cdot\left(12 \sqrt{f_{1}\left(\eta, c_{2}\right) f_{2}\left(\eta, c_{2}\right)}\right)^{-1}\right) d \eta
\end{aligned}
$$$$
=\int_{0}^{M\left(c_{1}\right)} \eta \frac{\left(12+\beta^{2}\right)\left(c_{2}-c_{1}\right)}{12 \sqrt{f_{1}\left(\eta, c_{2}\right) f_{2}\left(\eta, c_{2}\right)}} d \eta>0 .
$$

Combining Lemmas 7 and 8, we obtain $d^{\prime \prime}(c)>0$. The proof of Theorem 5 is completed.

\section{Conflict of Interests}

The author declares that there is no conflict of interests regarding the publication of this paper.

\section{Acknowledgments}

This work is supported by the National Natural Science Foundations of China (Grant nos. 11326123 and 11401096) and Guangdong Provence (no. GDJG20141204). The authors thank the editors for their hard work and also gratefully acknowledge helpful comments and suggestions by reviewers.

\section{References}

[1] A. Constantin and D. Lannes, "The hydrodynamical relevance of the Camassa-Holm and Degasperis-Procesi equations," Archive for Rational Mechanics and Analysis, vol. 192, no. 1, pp. 165-186, 2009.

[2] A. Geyer, "Solitary traveling water waves of moderate amplitude," Journal of Nonlinear Mathematical Physics, vol. 19, supplement 1, pp. 104-115, 2012.

[3] R. S. Johnson, "Camassa-Holm, Korteweg-de Vries and related models for water waves," Journal of Fluid Mechanics, vol. 455, pp. 63-82, 2002.

[4] R. Camassa and D. D. Holm, "An integrable shallow water equation with peaked solitons," Physical Review Letters, vol. 71, no. 11, pp. 1661-1664, 1993.

[5] A. Constantin, "On the scattering problem for the CamassaHolm equation," Proceedings of the Royal Society of London A: Mathematical, Physical and Engineering Sciences, vol. 457, pp. 953-970, 2001.

[6] A. Constantin, V. S. Gerdjikov, and R. I. Ivanov, "Inverse scattering transform for the Camassa-Holm equation," Inverse Problems, vol. 22, no. 6, pp. 2197-2207, 2006.

[7] A. Boutet de Monvel, A. Kostenko, D. Shepelsky, and G. Teschl, "Long-time asymptotics for the Camassa-Holm equation," SIAM Journal on Mathematical Analysis, vol. 41, no. 4, pp. 15591588, 2009. 
[8] K. El Dika and L. Molinet, "Exponential decay of $H^{1}$-localized solutions and stability of the train of $N$ solitary waves for the Camassa-Holm equation," Philosophical Transactions of the Royal Society of London Series A, vol. 365, no. 1858, pp. 23132331, 2007.

[9] Z. R. Liu and T. F. Qian, "Peakons of the Camassa-Holm equation," Applied Mathematical Modelling, vol. 26, no. 3, pp. 473-480, 2002.

[10] W. L. Zhang, "General expressions of peaked travelling wave solutions of $\mathrm{CH}-\gamma$ and $\mathrm{CH}$ equations," Science in China Series A: Mathematics, vol. 47, no. 6, pp. 862-873, 2004.

[11] A. Bressan and A. Constantin, "Global conservative solutions of the Camassa-Holm equation," Archive for Rational Mechanics and Analysis, vol. 183, no. 2, pp. 215-239, 2007.

[12] A. Constantin and J. Escher, "Analyticity of periodic traveling free surface water waves with vorticity," Annals of Mathematics, vol. 173, no. 1, pp. 559-568, 2011.

[13] A. Constantin and J. Escher, "Wave breaking for nonlinear nonlocal shallow water equations," Acta Mathematica, vol. 181, no. 2, pp. 229-243, 1998.

[14] A. Constantin and L. Molinet, "Global weak solutions for a shallow water equation," Communications in Mathematical Physics, vol. 211, no. 1, pp. 45-61, 2000.

[15] A. Bressan and A. Constantin, "Global dissipative solutions of the Camassa-Holm equation," Analysis and Applications, vol. 5, no. 1, pp. 1-27, 2007.

[16] A. Constantin and W. A. Strauss, "Stability of peakons," Communications on Pure and Applied Mathematics, vol. 53, no. 5, pp. 603-610, 2000.

[17] A. Constantin and W. A. Strauss, "Stability of the CamassaHolm solitons," Journal of Nonlinear Science, vol. 12, no. 4, pp. 415-422, 2002.

[18] Z. Y. Ouyang, S. Zheng, and Z. R. Liu, "Orbital stability of peakons with nonvanishing boundary for $\mathrm{CH}$ and $\mathrm{CH}$ jequations," Physics Letters A, vol. 372, no. 47, pp. 7046-7050, 2008.

[19] T. B. Benjamin, “The stability of solitary waves," Proceedings of the Royal Society -London Series A, vol. 328, pp. 153-183, 1972.

[20] K. El Dika and L. Molinet, "Exponential decay of $H^{1}$-localized solutions and stability of the train of $N$ solitary waves for the Camassa-Holm equation," Philosophical Transactions of the Royal Society of London A: Mathematical, Physical and Engineering Sciences, vol. 365, no. 1858, pp. 2313-2331, 2007.

[21] K. El Dika and L. Molinet, "Stability of multipeakons," Annales de l'Institut Henri Poincaré (C) Analyse Non Linéaire, vol. 26, no. 4, pp. 1517-1532, 2009.

[22] M. Grillakis, J. Shatah, and W. Strauss, "Stability theory of solitary waves in the presence of symmetry I," Journal of Functional Analysis, vol. 74, no. 1, pp. 160-197, 1987.

[23] N. DurukMutlubas and A. Geyer, "Orbital stability of solitary waves of moderate amplitude in shallow water," Journal of Differential Equations, vol. 255, no. 2, pp. 254-263, 2013.

[24] N. D. Mutlubaş, "On the Cauchy problem for a model equation for shallow water waves of moderate amplitude," Nonlinear Analysis: Real World Applications, vol. 14, no. 5, pp. 2022-2026, 2013. 


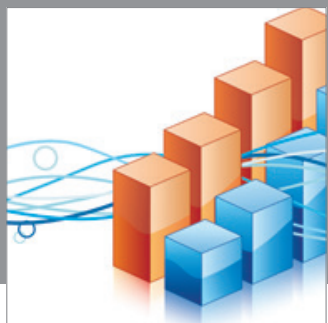

Advances in

Operations Research

mansans

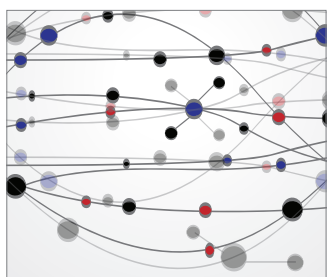

The Scientific World Journal
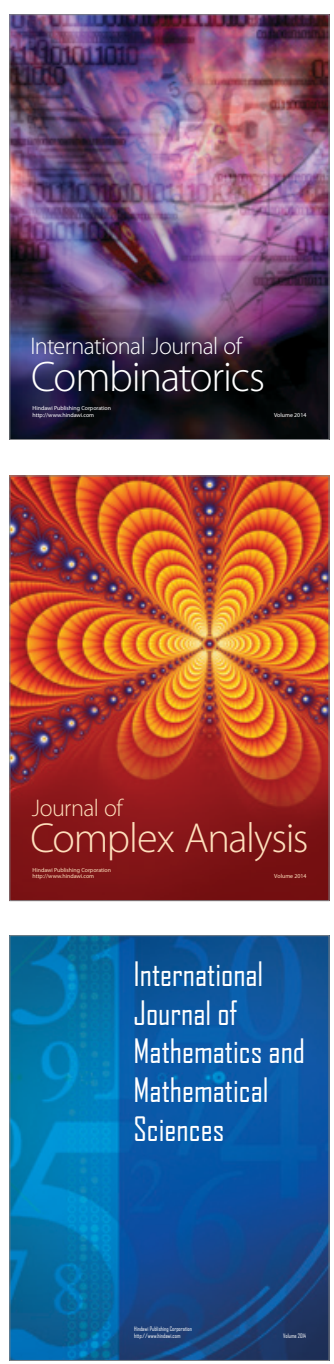
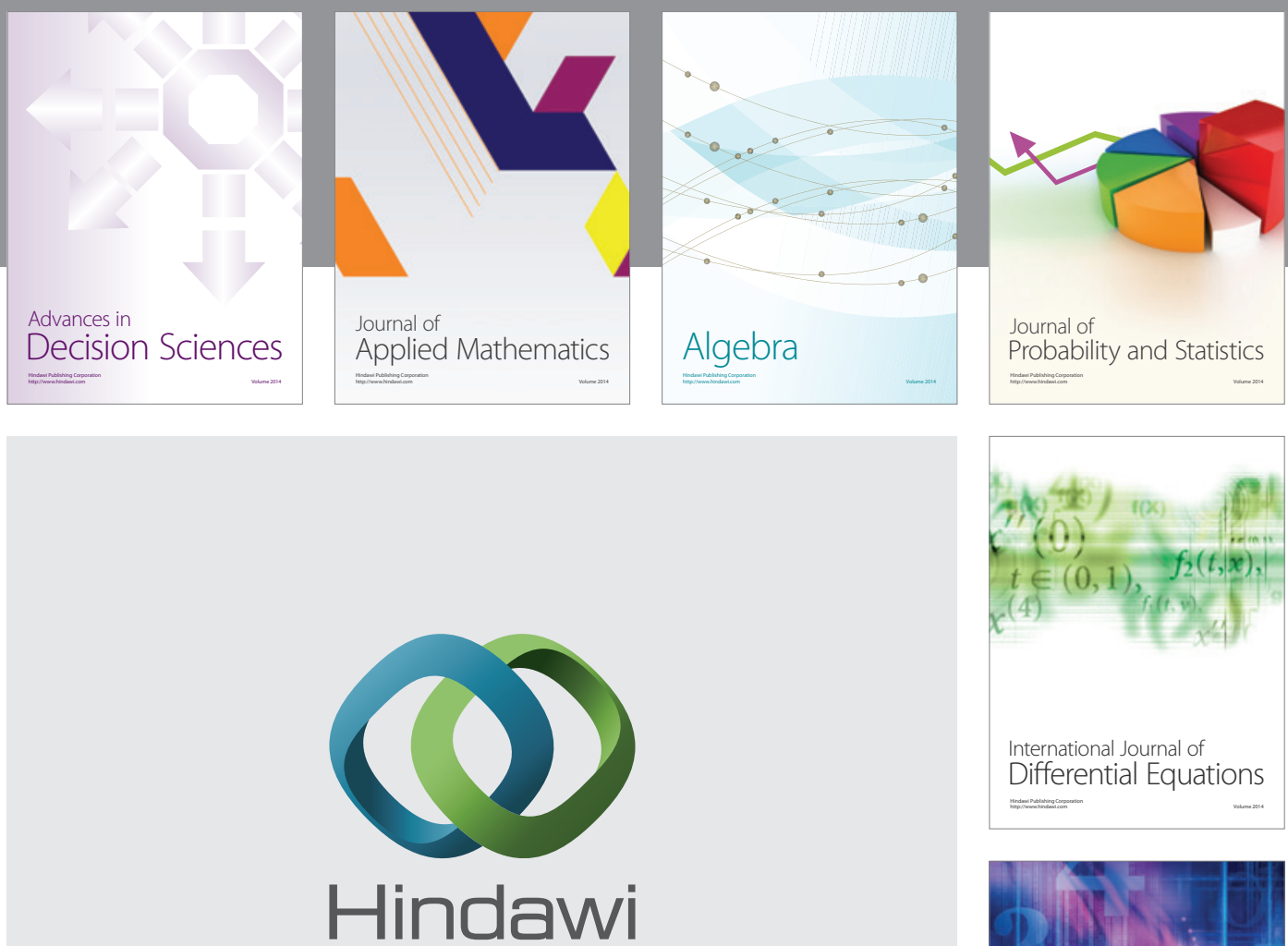

Submit your manuscripts at http://www.hindawi.com
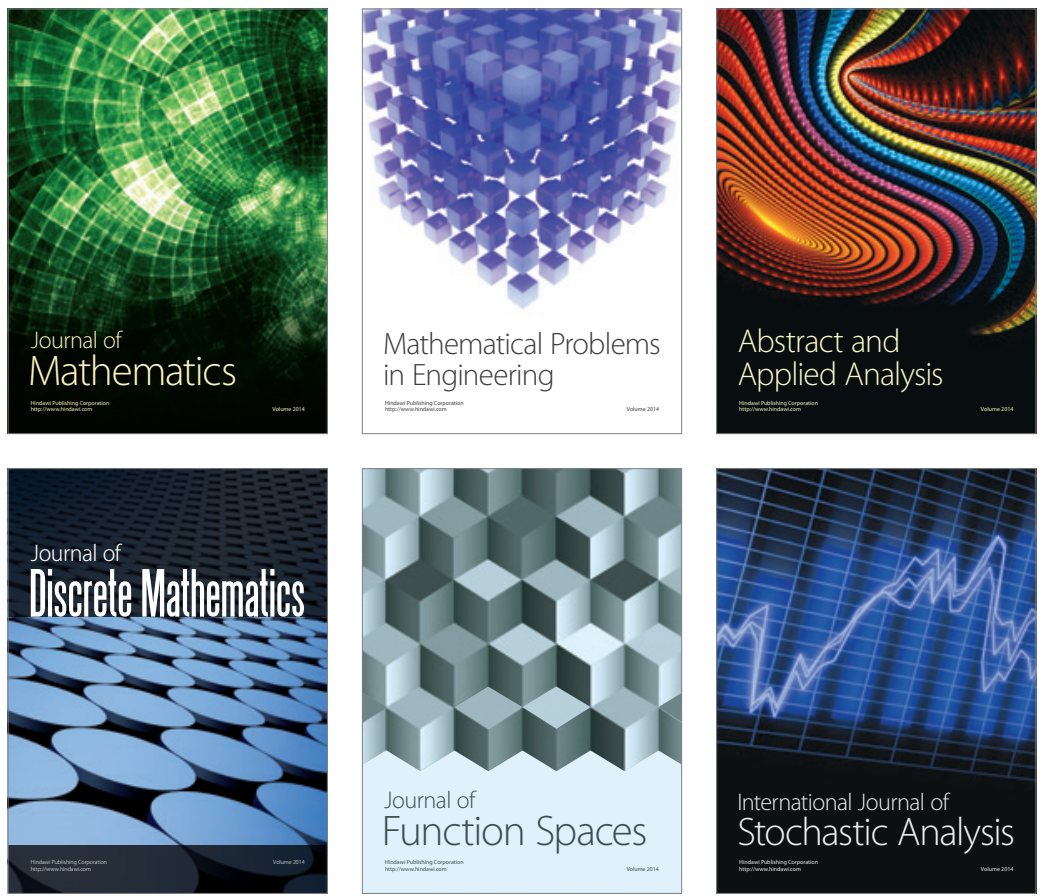

Journal of

Function Spaces

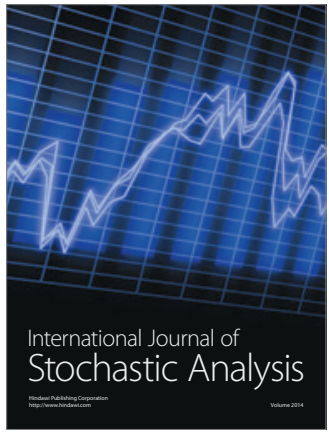

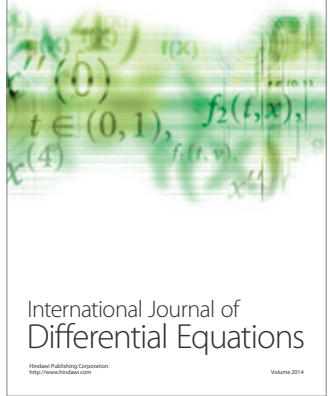
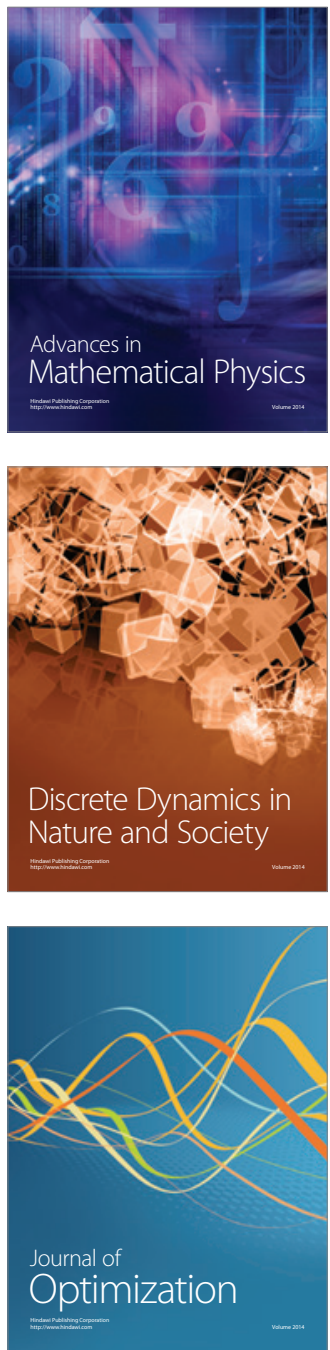